\title{
Mapping magnetism onto the core
}

Telling how the Earth's molten core generates the terrestrial magnetic field requires downward projection of the measured surface field, which is not as simple as it may seem.

IF the Earth's magnetism stems from what happens in the molten core, then it should be possible to tell what happens in the core by careful observation and analysis of the magnetic field on and above the surface of the Earth. Nobody will dissent from that tautology. The difficulties arise only in the practice of this problem in inversion, but the result is that, while much has been written on the subject, little is known about the pattern of the Earth's magnetism at the boundary between the core and the mantle.

As in other problems of this kind, there is a competition between the improved accuracy of the data now available, especially from satellite measurements, and the need which they compel to take account of complicating refinements in the analysis. This is the spirit in which Andrew Jackson from Harvard University has set out to estimate the extent to which inferences about the magnetic field at the surface of the molten core must be corrected for the magnetization of the crustal rocks (Geophys. J. Int. 103, 657; 1990).

The standard treatment of the problem, due to Gauss at the beginning of the nineteenth century, is to represent the measured magnetic field at the surface of the Earth by a sum of spherical harmonic functions, which do for the surface of a sphere what the sine and cosine functions do for the circumference of a circle, and which are mutually orthogonal when integrated over a sphere. Such a representation of the surface field is then also a valid basis for the representation of the magnetic field in any contiguous region free from electric current, which is probably a fair assumption all the way down to the core-mantle boundary. That is how this representation can be a way of mapping the surface field onto the external surface of the core.

One practical snag is that this description of the Earth's measured magnetic field at the surface is always incomplete. The spherical harmonics are, as they must be, an infinite set of functions, categorized by an integer which represents the degree of the polynomials in the trigonometric functions of the latitude appearing in the spherical harmonic function. The simplest of these represents a dipole field, but the Earth's field is not a simple dipole, which makes it necessary to include spherical harmonics of higher degree.

Evidently, features such as the magnetic anomaly in the southwest Atlantic, an apparent patch of abnormally low magnetic field, require that harmonic functions of high degree should be included. A few years ago, people were using combinations of spherical harmonics up to degree ten. Now, with the satellite data, truncation is not required by the inaccuracy of the data until degree 60 or more.

The practical problem that arises is that these functions, when used for downward mapping into the Earth, also depend on the radius by an inverse power which is essentially the degree of the spherical harmonic. This implies that minor errors of representation at the surface may be very large when extrapolated downwards to the core, perhaps in the ratio $(6,371 / 3,485)^{n}$, where the numerator and denominator are the radii of the surface and the core (in kilometres) respectively and $n$ is the degree of the spherical harmonic.

That is a familiar hazard of downward mapping. Jackson is concerned with a more subtle source of difficulty - the extent to which the magnetization of the material of the Earth may spuriously contribute to measurements of the nearsurface field. If, of course, the material of the Earth were uniform in its magnetic properties, and if its magnetiation were simply determined by the local field, the inversion problem would not be further complicated. But the Earth is plainly far from uniform magnetically.

By Jackson's account, this complication presents itself even in simple-minded attempts to represent the measured field by spherical harmonic functions of evergreater degree. If the underlying dynamo embedded in the core is a reasonably simple structure, one would expect that the importance in the surface representation of the field of particular spherical harmonics would diminish as the degree increases. Ten years or so ago, with the data then available, that expectation seemed to be fulfilled. But now, with data from satellite measurements, it seems that the importance of spherical harmonics of higher degree decreases only up to degree 15 or thereabouts, after which it increases again.

Plainly this is not merely an invitation to disaster in the downward mapping of the surface field, but it also raises the physical question of what can account for such small-scale structure in the pattern of the surface field.

Crustal magnetism seems to be the explanation, but Jackson also quotes an interesting argument due to G. E. Backus to show that the higher harmonics cannot originate in the core. It is simply that if these components of the field were to originate in currents in the core, those same currents would occasion Ohmic heating which could not in sum exceed the measured output of heat from the surface of the Earth. This allows him to say with some conviction that the harmonics of degree greater than 25 or thereabouts must certainly have their origin outside the core.

Jackson allows that the importance of the contamination of the surface measurements by the magnetization of the surface rocks has been determined by others. All of us can think of ways in which this contamination may come about. Masses of igneous rock may be magnetized in the direction of the Earth's field when they were first formed while, on the ocean floor, there are the well-known magnetic stripes used to determine the rate of seafloor spreading. Jackson's chief purpose is to find some way of removing the contamination statistically.

There seem to be two extreme models of the contaminating field. One is to suppose that there are regional variations of magnetic susceptibility, and thus of induced magnetism, and to construct a model of the field from geological data. At the other extreme is the supposition that the continents and the sea-floor are separately homogenous, but markedly different from each other in magnetic properties. For what it is worth, Jackson comes down marginally against the second model.

But the nub of his conclusion is surprising. On the assumption that the magnetic properties of the surface rocks are spatially random, a kind of white noise, he is nevertheless able to show that there will be correlations in the induced magnetization over short length-scales, perhaps even as great as 15 degrees on the surface of the Earth. This, he argues, is a good starting-point in a search for an explanation of the higher-order harmonics in the representation of the surface field. Further refinements could be brought about by allowing for the magnetic stripes on the ocean floors as well as other obvious departures from uniformity. The next step will be numerical calculation of what the corrected field is like at the surface of the core. The outcome could be more entertaining than many numerical calculations.

John Maddox 